L. L. Avramov

Nagoya Math. J.

Vol. 88 (1982), 161-180

\title{
PSEUDO-REFLECTION GROUP ACTIONS ON LOCAL RINGS
}

\author{
LUCHEZAR L. AVRAMOV
}

\section{§1. Introduction}

In a classical paper [C] Chevalley considered the invariants of a finite group $H \subset G L_{k}\left(S_{1}\right)$ generated by pseudo-reflections, acting on the graded polynomial ring $S=k\left[X_{1}, \cdots, X_{n}\right]$ over a field $k$ of characteristic zero. He proved that $S$ is free as a graded $S^{H}$-module, hence $S^{H}$ is a graded polynomial ring (Theorem A), and that the natural representation of $H$ in $S / S_{+}^{H} S$ is equivalent to the regular representation (Theorem $\mathrm{B}$ ). On the other hand, a theorem of Shephard and Todd shows that when $S^{H}$ is a polynomial ring, the (finite) group $H$ is generated by pseudo-reflections. These results have been extended by Bourbaki $\left[\mathrm{Bo}_{2}\right]$ to fields whose characteristic may be positive, but does not divide the order $|H|$ of the group.

The study of invariants of pseudo-reflection groups acting on local rings was initiated and largely motivated by Serre's [Se] generalization of the Chevalley-Shephard-Todd theorem to regular local rings. In this note we are interested in a general local situation. To describe it we introduce some notation which will be in force for the rest of the paper. We fix a commutative noetherian unitary local ring $(R, \mathfrak{m}, k)$, and a finite group $G$ of automorphisms of $R$. The subring of invariants $R^{a}=\{x \in R \mid \forall g \in G: g(x)$ $=x\}$, over which $R$ is integral, is local with maximal ideal $\mathfrak{m}^{G}=\mathfrak{m} \cap R^{G}$ (cf. e.g. $\left.\left[\mathrm{Bo}_{1}\right]\right)$. For an ideal $\mathfrak{a} \subset R, G^{T}(\mathfrak{a})=\{g \in G \mid \forall x \in R: g(x)-x \in \mathfrak{a}\}$ is the inertia subgroup of $a$. We set $H=G^{T}(\mathfrak{m})$, which is the normal subgroup of $G$, equal to $\operatorname{Ker}(G \rightarrow \operatorname{Aut}(k))$, and denote by $\varepsilon$ the canonical homomorphism $H \rightarrow G L_{k}\left(\mathrm{~m} / \mathrm{m}^{2}\right)$. When $|H| \in R^{\times}$(= the group of units of $R$ ), $R^{G}$ is noetherian, $R^{G} / \mathfrak{m}^{G}=k^{G / H}$, and $\varepsilon$ is injective: cf. (8), (9), and (10) below. An element $h \in H$ is called a pseudo-reflection when $\varepsilon(h)$ is a pseudo-reflection in the sense of linear algebra, i.e. when $\operatorname{rank}(\varepsilon(h)-1)=1$. From lemma (12) below one has:

Received December 22, 1980.

Revised May 12, 1982. 
(1) Suppose $|H| \in R^{\times}$. Then $h \in H$ is a pseudo-reflection if and only if there exists an element $x_{h} \in \mathfrak{m}$ such that $h \in G^{T}\left(x_{h} R\right)$. In this case $x_{h} \notin \mathfrak{m}^{2}$, and is defined uniquely up to an invertible factor.

We say that the action of $G$ is generically without inertia if every associated prime of $R$ has a trivial inertia subgroup. Clearly, it suffices to impose the restriction on the maximal elements of Ass $(R)$, and the condition is trivially satisfied when $R$ is a domain. The symbol $R * G$ is used to denote the skew group ring (or trivial crossed product), i.e. the free $R$-module with basis $g \in G$ and (non-commutative when $G \neq 1$ ) multiplication defined by $\left(x_{1} g_{1}\right)\left(x_{2} g_{2}\right)=x_{1} g_{1}\left(x_{2}\right) g_{1} g_{2}$. In order to avoid confusion we shall, for $a=\sum_{g \in G} a_{g} g \in R * G$ and $x \in R$, write $a x$ when the product is taken in $R * G$, and $a(x)$ when it is in the $R * G$-module $R: a(x)=\sum_{g \in G} a_{g} g(x)$. Finally, the group ring of $G$ over $R^{a}$ is denoted $R^{a}[G]$.

(2) Theorem. Suppose that $|H| \in R^{\times}$, and that generically $G$ acts on $R$ without inertia. If $H$ is generated by pseudo-reflections, then:

(i) $R$ has a normal basis with respect to $G$, i.e. $R \simeq R^{G}[G]$ as $R^{G}[G]-$ modules; in particular:

(i) $\quad R$ is a free $R^{a}$-module of rank $|G|$;

(ii) the fibre $(\bar{R}, \overline{\mathfrak{m}})=\left(R / \mathfrak{m}^{G} R, \mathfrak{m} / \mathfrak{m}^{G} R\right)$ is an (artinian) strict complete intersection, in the sense that the associated graded ring $\operatorname{gr}_{\overline{\mathrm{m}}} \bar{R}$ also is a complete intersection.

Recall that the complete intersection defect, or deviation, of $R$ is defined to be the integer $d(R)=\nu(a)-(\operatorname{dim} \tilde{R}-\operatorname{dim} R)$, where $\tilde{R}$ is some regular local ring, provided by Cohen's structure theorem, such that $\hat{R}=\tilde{R} / a$, and $\nu$ denotes the minimal number of generators; this is an invariant of $R$, and $d(R) \geqslant 0$, with equality holding precisely for the complete intersections. When $R$ is Cohen-Macaulay, the type of $R$ is by definition the integer $t(R)$ $=\operatorname{dim}_{k} \operatorname{Ext}_{R}^{\mathrm{dim} R}(k, R)$; then $t(R) \geqslant 1$, the equality characterizing the Gorenstein rings. The Hilbert-Samuel series of $R$ is the (rational) power series $H_{R}^{(1)}(t)=\sum_{n \geqslant 0} \ell_{R}\left(R / \mathfrak{m}^{n+1}\right) t^{n}$; the function $n \mapsto \ell_{R}\left(R / \mathfrak{m}^{n+1}\right)$ is a polynomial for sufficiently large $n$, and $(\operatorname{dim} R)$ ! times its leading coefficient is the multiplicity $e(R)$. The Poincaré series of a finitely-generated $R$-module $M$ is the formal power series $P_{R}^{M}(t)=\sum_{n \geqslant 0} \operatorname{dim}_{k} \operatorname{Tor}_{n}^{R}(k, M) t^{n}$. The sign $\ll$ denotes a coefficientwise inequality of formal power series.

(3) CoRollary. Under the assumptions of (2) one has:

(i ) $d\left(R^{G}\right)=d(R)$; in particular, $R^{G}$ is a complete intersection if and only 
if this is true for $R$;

(ii) $R^{G}$ is Cohen-Macaulay of type $t$ if and only if this is true for $R$; in particular, $R^{G}$ is Gorenstein if and only if $R$ is Gorenstein;

(iii) for every finitely-generated $R * G$-module $M, M^{G}$ is a finitely-generated $R^{G}$-module and

$$
P_{R^{G}}^{M^{G}}(t) \ll P_{R}^{M}(t), \quad P_{R^{G}}^{k^{G}}(t)=P_{R}^{k}(t) \cdot(1-t)^{m},
$$

where $m$ is the difference of the embedding dimensions of $R$ and $R^{a}$;

(iv) $H_{R^{G}}^{(1)}(t) \ll H_{R}^{(1)}(t)$, hence $e\left(R^{G}\right) \leqslant e(R)$;

(v) if $R$ is a normal domain, then so is $R^{G}$, and the canonical map of divisor class groups $C l\left(R^{a}\right) \rightarrow C l(R)$ is an injection; in particular, if $R$ is factorial, then $R^{G}$ is factorial.

The references are: [A, (3.6)] for (i); [HK, (1.24)] for (ii); $\left[\mathrm{G}_{2},(1.1)\right]$, applied via (8) and (10) below-for the inequality in (iii); [A, (1.1)] for the equality in (iii); [L, Remark 4, p. 87] for (iv); [F, (6.11)] for (v).

Recall that a prime $\mathfrak{B} \subset R$ is said to be unramified over $R^{G}$ if for $\mathfrak{p}=$ $\mathfrak{P} \cap R^{G}$ the field extension $R_{\mathfrak{p}}^{G} / \mathfrak{p} R_{\mathfrak{p}}^{G} \hookrightarrow R_{\mathfrak{\beta}} / \mathfrak{\beta} R_{\mathfrak{\beta}}$ is separable, and $\mathfrak{p} R_{\mathfrak{\Re}}=\mathfrak{\beta} R_{\mathfrak{\beta}}$; otherwise $\mathfrak{P}$ is said to be ramified. The ramification locus of $R$ over $R^{G}$ is the closed subset of Spec $R$, consisting of primes containing one of the three differents: the Dedekind, Noether, and Kähler one (for the notions of ramification theory used in this paper we refer to the lecture notes of Scheja and Storch $\left.\left[\mathrm{SS}_{1}\right]\right)$. According to Theorem (2), we are under the hypotheses of $\left[\mathrm{SS}_{1},(16.8)\right]$ and of $\left[\mathrm{SS}_{2},(5.5),(5.6)\right]$, which show that all three differents of $R$ over $R^{G}$ coincide, and are a principal ideal, denoted henceforth by $\mathfrak{D}\left(R \mid R^{G}\right)$. Our main result in this note is the complete description of the ramification of $R$ over $R^{G}$.

(4) Theorem. Suppose $G$ acts on $R$ generically without inertia, $H$ is generated by pseudo-reflections, and $|H| \in R^{\times}$. Let $h_{1}, \cdots, h_{r}$ be all the distinct pseudo-reflections contained in $H$, and let $x_{i}$ be an element defined by $h_{i}$ according to (1).

Then $\mathfrak{S}\left(R \mid R^{G}\right)$ is the regular principal ideal of $R$ generated by $x=$ $\prod_{i=1}^{r} x_{i}$, and the discriminant $\mathfrak{b}\left(R \mid R^{G}\right)$ is the regular principal ideal of $R^{G}$ generated by $N_{G}(x)=\prod_{g \in G} g(x)$.

Moreover, $\mathfrak{S}\left(R \mid R^{G}\right)$ is equal to the intersection of all $H$-stable ideals of $R$, which are not contained in $\mathfrak{m}^{G} R=\mathfrak{m}^{H} R$.

(5) Remarks. We assume for simplicity $G=H$, and $|H| \in R^{\times}$. 
(i) After Serre proved [Se] (cf. also the proofs in [ $\left[\mathrm{Bo}_{2}\right.$, Exercise 7], [St, (3.7)], [Wj, Section 2]) that the descent of regularity from $R$ to $R^{H}$ is equivalent to the fact that $H$ is generated by pseudo-reflections, various properties of $R$ have been shown to be inherited by $R^{H}$ under this assumption on the group. Under slightly restrictive conditions on $R$, this was obtained in $\left[\mathrm{Si}_{1}\right]$ for unique factorization; (3.v) extends and generalizes his result, and is itself contained in a theorem of Storch [St, (3.9)], which holds for more general groups. The claim of (3.ii) is a more precise version of a result obtained by Hochster and Eagon for graded rings [HE, Proposition 15]. In one direction, (3.ii) holds in the Gorenstein case without assumptions on the action of $G: \mathrm{K}$. Watanabe has shown $\left[\mathrm{Wk}_{2}\right.$ ] that $\mathrm{R}$ Gorenstein implies $R^{G}$ Gorenstein. A partial generalization of this has been given by Goto $\left[G_{1}\right],\left[G_{2}\right]$, assuming the action of $G$ to be liftable to a regular local ring; in the same situation, he also shows that $d\left(R^{a}\right) \leqslant d(R)$. In view of these results, and (3.i), (3.ii) above, it is natural to ask the

Question. Assume $|H| \in R^{\times}$and $H$ is generated by pseudo-reflections. Is it true that $d\left(R^{G}\right) \leqslant d(R)$, and when $R$ is Cohen-Macaulay, that $t\left(R^{G}\right) \leqslant$ $t(R) ?^{(*)}$

(ii) Chevalley's Theorem B [C] has been extended in $\left[\mathrm{G}_{2}(1.2)\right]$ to local domains. Theorem (2.i) is a further generalization, allowing some zerodivisors.

(iii) Singh has recently asked whether the inequality $H_{R^{(}}^{(0)}(t) \ll H_{R}^{(0)}(t)$ holds for the Hilbert series $H^{(0)}(t)=(1-t) \cdot H^{(1)}(t)\left[\mathrm{Si}_{2}\right.$, Question 1]; assuming the action of $G$ is liftable to a regular local ring, he has answered in the affirmative when $R$ is a hypersurface ring or when $G$ is abelian. Our result in (3.iv) can be viewed as a strong argument in favor of the conjecture.

(iv) The description of the different given in Theorem (4) has a classical counterpart in the case of pseudo-reflection group actions on polynomial rings: cf. [ $\mathrm{Bo}_{2}$, Proposition 6.(i)].

(v) Although the assumption that $G$ acts on $R$ without generic inertia is not necessary for the $R^{G}$-freeness of $R$, it is seen from (6) that it is implied by the conclusions of $\left(2 . i^{\prime}\right)$ and of (4).

(*) The inequality of the types has been meanwhile proved by A. Aramova in her M. A. thesis (University of Sofia, 1981). On the other hand, using G. Müller's result (20) as in Step 3 of the proof of Theorem (17) in Section 6, one can deduce in the equicharacteristic case both inequalities from the particular case proved in $\left[\mathrm{G}_{1}\right]$. 
(vi) When $H$ is generated by generalized reflections, i.e. by elements lying in the inertia subgroups of regular principal ideals (cf. [HE]), the $R^{H}$-flatness of $R$ is known (cf. (19) below); combined with the finiteness result in (8), this implies freeness. Since it is easily seen (cf. (18.ii)) that under the hypotheses of (2) $H$ is generated by generalized reflections, the freeness statement in $\left(2 . \mathrm{i}^{\prime}\right)$ (but not the information on the rank, when $R$ has zero-divisors), can be alternately deduced from the existing literature. Thus it seems interesting to compare the hypotheses of our main results (2) and (4) to the seemingly weaker requirement that $H$ be generated by generalized reflections (which is the case considered by several authors, e.g. [HE], [Wj], $\left.\left[\mathrm{Si}_{2}\right]\right)$. It is the subject of Section 6 , to show that-at least under equicharacteristic assumptions, and presumably in general-the hypotheses are equivalent. The proof of this result, Theorem (17), depends on (2) and (4).

Theorems (2) and (4) are proved in sections 4 and 5 respectively. In the next section we establish several results of a general nature, some of them interesting in their own right, while section 3 contains preliminaries on pseudo-reflections.

\section{§2. Finite group actions}

The notation used below is that of the Introduction. The assumptions on $R, G$, etc. are detailed in each statement.

(6) Let $\mathfrak{B}_{1}, \cdots, \mathfrak{P}_{s}$ be a set of prime ideals of $R$, such that the multiplicatively closed set $U=R \backslash \bigcup_{i=1}^{s} \mathfrak{P}_{i}$ is G-stable. With $N_{G}(u)=\prod_{g \in G} g(u)$, set $V=\left\{N_{G}(u) \mid u \in U\right\}$.

Then $V^{-1} R=U^{-1} R, V^{-1}\left(R^{G}\right)=\left(U^{-1} R\right)^{G}$, and the following conditions are equivalent:

(a) $G^{T}\left(\mathfrak{P}_{i}\right)=1$ for $1 \leqslant i \leqslant s$;

(b) $U^{-1} R$ is unramified over $V^{-1} R^{a}$, and is free of rank $|G|$ as a $V^{-1} R^{G}$ module.

When these conditions are fulfilled, $U^{-1} R \simeq\left(V^{-1} R^{G}\right)[G]$ as $\left(V^{-1} R^{G}\right)[G]-$ modules.

For the isomorphism of $V^{-1} R^{G}$ and $\left(U^{-1} R\right)^{G}$ cf. e.g. [SS, p. 104]. In particular, it shows that $U^{-1} R$ is integral over $V^{-1} R^{G}$, hence the last ring is semi-local. Note that the maximal ideals of $U^{-1} R$ are among the $U^{-1} \Re_{i}$ $(1 \leqslant i \leqslant s)$, and that $G^{T}\left(\mathfrak{P}_{i}\right)=G^{T}\left(U^{-1} \mathfrak{P}_{i}\right)$ by $\left[\mathrm{Bo}_{1}\right.$, Lemma 3]. The equivalence 
of (a) and (b) becomes now a particular case of $\left[\mathrm{SS}_{1},(21.7)\right]$. (An alternate reference for the implication $(\mathrm{a}) \Rightarrow(\mathrm{b})$ is given by [CHR, (1.3) and (4.1)].) The last statement is obtained from [CHR, (4.2.c)].

The first statement of the following proposition is taken from $\left[\mathrm{SS}_{1}\right.$, (20.4)], while the second one comes from [CHR, (1.6)].

(7) $R^{H}$ is unramified over $R^{G}$, and is a free $R^{G}$-module of rank $|G / H|$. There exists an element $r^{\prime} \in R^{H}$, such that $t_{G / H}\left(r^{\prime}\right)=1 \in R^{G}$.

Here and below $t_{G}$ denotes the trace element $\sum_{g \in G} g \in R * G$.

(8) Suppose $|H| \in R^{\times}$. Then:

(i) $R^{G}$ is a noetherian local ring;

(ii) an $R$-module $M$ is finitely-generated over $R$ (if and) only if it is finitely-generated over $R^{G}$.

Proof. That $R^{G}$ is local is an immediate consequence of the fact that $R$ is integral over it. That it is noetherian can either be deduced from (ii) via the Eakin-Nagata theorem, or seen directly as follows: the noetherian property descends from $R$ to $R^{H}$ because of the existence of the Reynolds operator $|H|^{-1} t_{H}: R \rightarrow R^{H}$, and from $R^{H}$ to $R^{G}$ by faithful flatness (7).

As for (ii), it is sufficient to prove that $R$ is finitely-generated over $R^{G}$, which follows from the finite generation of $R^{H}$ over $R^{G}$ (7), and that of $R$ over $R^{H}$ [LP, (3.4)].

(9) The following conditions are equivalent:

(a) $|H| \in R^{\times}$;

(b) ( $)^{G}$ is an exact functor from the category of left $R * G$-modules to that of $R^{G}$-modules;

(c) there exists an element $r \in R$ with $t_{G}(r)=1$.

When these conditions are satisfied, $M^{G}=t_{G}(M)$, and ()$^{G}$ carries finitelygenerated modules to finitely-generated ones.

Proof. The equivalence of (b) and (c) is established in $\left[G_{2},(2.2)\right]$ (cf. also [X, Lemma 1]).

Assume (c), and denote by $\bar{r}$ the image of $r$ in $k$. Then in $R^{G} / \mathfrak{m}^{G}$ one has:

$$
1=t_{G}(\bar{r})=t_{G / H}\left(t_{H}(\bar{r})\right)=|H| t_{G / H}(\bar{r}),
$$

hence $|H| \in\left(R^{G}\right)^{\times} \subset R^{\times}$, which is (a). 
Conversely, suppose (a) is satisfied, and let $r^{\prime} \in R^{H}$ be an element given by (7). Since $r^{\prime}=|H|^{-1} t_{H}\left(r^{\prime}\right)$, one has:

$$
1=t_{G / H}\left(r^{\prime}\right)=t_{G / H}\left(|H|^{-1} t_{H}\left(r^{\prime}\right)\right)=t_{G}\left(|H|^{-1} r^{\prime}\right),
$$

hence (c) holds.

For the last statement, note that $t_{G}(m)$ clearly belongs to $M^{G}$, while the inverse inclusion follows from the equalities $m^{\prime}=t_{G}(r) m^{\prime}=t_{G}\left(r m^{\prime}\right)$, holding when $m^{\prime} \in M^{a}$; the claim on finite generation is a consequence of (8.ii).

As an immediate consequence of the last two lemmas we have the next statements, of which (i) is well-known: cf. e.g. [ $\mathrm{Bo}_{2}$, Exercise 7(c)], [Si ${ }_{1}$, Lemma 3], [Wj, (2.16)], etc. The conclusion of (ii) is asserted in [K, Chapter II, Lemma 1] under the assumption that $R^{G}$ is noetherian; however, in the proof the finite generation of $R$ over $R^{G}$ is essentially used, and it is not clear whether it is implied by the noetherian character of $R^{G}$.

(10) Suppose $|H| \in R^{\times}$. Then one has:

(i) the natural map $\varepsilon: H \rightarrow G L_{k}\left(\mathrm{~m} / \mathrm{m}^{2}\right)$ is injective;

(ii) denoting by ( $)^{\wedge}$ completions in the respective maximal-ideal-adic topologies, $\hat{R} \simeq R \otimes_{R^{G}}\left(R^{G}\right)^{\wedge}$, hence $G$ acts naturally on $R$ via the first factor, and $(\hat{R})^{G} \simeq\left(R^{G}\right)^{\wedge}$.

We skip the proof of (i). The first isomorphism in (ii) is immediate from (8), since $\mathrm{m}^{G} R$ is an $m$-primary ideal, and it implies the second one by the flatness of the completion: cf. $\left[\mathrm{SS}_{1},(19.2)\right]$.

(11) Let $Q=\mathrm{gr}_{\mathrm{m}} R=\oplus_{i \geqslant 0} \mathrm{~m}^{i} / \mathrm{m}^{i+1}$, and let $S$ be the symmetric algebra over $k$ of $\mathrm{m} / \mathrm{m}^{2}$, considered with the natural degree-preserving actions of $H$ by k-algebra automorphisms, induced by $\varepsilon$.

If $|H| \in R^{\times}$, there exist natural $H$-equivariant surjective homomorphisms of graded k-algebras:

$$
\operatorname{gr}_{\overline{\mathrm{m}}} \bar{R} \longleftarrow Q / Q_{+}^{H} Q \longleftarrow S / S_{+}^{H} S
$$

where ( ) ${ }_{+}$denotes the irrelevant maximal ideal, and $\bar{R}=R / \mathfrak{m}^{G} R$.

Proof. By the universal property of the symmetric algebra, there is a surjective homomorphism $p: S \rightarrow Q$ of graded $k$-algebras, which in degree one is the identity map of $\mathrm{m} / \mathrm{m}^{2}$, and which defines a commutative diagram of homomorphisms of graded $k$-algebras: 


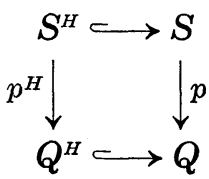

Note that by (9) $p^{H}$ also is surjective. This shows that with $k=S^{H} / S_{+}^{H}$, $Q \otimes_{S^{H}} k=Q / S_{+}^{H} Q=Q / Q_{+}^{H} Q$, hence the right-hand map is simply $p \otimes_{S^{H}} k$.

On the other hand, it is easy to see that $Q_{+}^{H}$ is contained in $I=\operatorname{Ker}(Q$ $\left.=\mathrm{gr}_{\mathrm{m}} R \rightarrow \mathrm{gr}_{\mathrm{m}} \bar{R}\right)$. In fact, suppose $a^{*} \in Q_{+}^{H}$ is the initial form in $Q$ of the element $a \in R$, such that $a \in \mathfrak{m}^{n} \mid \mathfrak{m}^{n+1}$. Then for every $g \in H$ one has $a-$ $g(a) \in \mathfrak{m}^{n+1}$, hence $a^{*}=|H|^{-1} \sum_{g \in H} g(a) \in I$. The inclusion of ideals $Q_{+}^{H} Q \in I$ defines the left-hand map. (The author is indebted to the referee for pointing out that the original longer proof of this inclusion could be replaced by the argument above.)

The $H$-equivariance of both maps is clear from their definition.

\section{§3. Pseudo-reflections}

In this section we assume that $|H| \in R^{\times}$. The equivalence of (a) and (b) in the first lemma has been proved under different restrictive assumptions in [Si, Lemma 6], and [Wj, (2.15)].

(12) The following conditions on $h \in H$ are equivalent:

(a) $h$ is a pseudo-reflection;

(b) $h \neq 1$, and there exists an element $x_{h} \in \mathfrak{m}$, such that $h \in H^{T}\left(x_{h} R\right)$;

(c) the ideal $a_{h}$, generated by all the elements $h(a)-a$ when a runs through $R$, is principal and different from zero.

Furthermore, when they are fulfilled, an element $x^{\prime} \in \mathfrak{m}$ has the property that $h \in H^{T}\left(x^{\prime} R\right)$ if and only if $x^{\prime}$ is a generator of $a_{h}$. In particular, $x^{\prime} \notin$ $\mathrm{m}^{2}$, and is defined uniquely up to an invertible factor.

Proof. (a) $\Rightarrow(\mathrm{c})$ : Suppose first $R$ is complete. In an appropriate basis of $\mathfrak{m} / \mathfrak{m}^{2}, \varepsilon(h)$ is given by a diagonal matrix, whose only entry different from 1 is a primitive $m$-th root of unity $\bar{\omega} \in k, m$ denoting the order of $h$ (cf. (10.i)). By Hensel's lemma and the condition that the characteristic of $k$ does not divide $|H|, R$ contains a unique primitive $m$-th root of unity $\omega$ which maps onto $\bar{\omega}$. Under these assumptions it is proved in [Se, pp. 8-9], or [ $\left[\mathrm{Si}_{1}\right.$, Lemma 6], that there exists a $x_{h} \in \mathfrak{m}$, such that $h\left(x_{h}\right)=\omega x_{h}$, $h \in H^{T}\left(x_{h} R\right)$. It is clear that $a_{h} \subset x_{h} R$, and the reverse inclusion is given by the equality $(\omega-1) x_{h}=h\left(x_{h}\right)-x_{h}$, since $\omega \neq 1$ modulo $\mathrm{m}$. 
Passing to the general case, we denote by $\mathfrak{a}_{h}$ the ideal defined in (c) for $R$, and by $\mathfrak{b}_{n}$ the corresponding one for $\hat{R}$. Let $b_{1}, \cdots, b_{q}$ be a system of generators (8.ii) of $R$ over $R^{(h)}$. According to (10.ii) they also generate $\hat{R}$ over $\hat{R}^{(h)}$. For an arbitrary $b \in \hat{R}$, write $b=\sum b_{i} c_{i}$ with $c_{i}$ in $\hat{R}^{(h)}$. Then $h(b)-b=\sum\left(h\left(b_{i}\right)-b_{i}\right) c_{i}$, hence $\mathfrak{b}_{h} \subset \mathfrak{a}_{h} \hat{R}$. The converse inclusion being obvious, we see from the first part that $\mathfrak{a}_{h} \hat{R}$ is principal, hence $a_{h}$ is principal.

(c) $\Rightarrow(b):$ Any generator of $a_{h}$ has the required properties, since $h \in H$ implies $\mathfrak{a}_{h} \subset \mathfrak{m}$.

(b) $\Rightarrow$ (a): Let $\bar{x}_{h}$ denote the image of $x_{h}$ in $\mathfrak{m} / \mathfrak{m}^{2}$. Since $W=\operatorname{Im}(\varepsilon(h)$ $-1) \subset \bar{x}_{h} k$, it is sufficient to show $W \neq 0$. But $W=0$ means $\varepsilon(h)=1$, hence by (10.i) $h=1$, which is absurd.

Let now $x^{\prime}$ be an element such that $h \in H^{T}\left(x^{\prime} R\right)$. The last part of the proof shows that $x^{\prime} \notin \mathrm{m}^{2}$. The ideal $x^{\prime} R$ being $h$-stable, $h\left(x^{\prime}\right)=c x^{\prime}$ for some $c \in R$, hence $\varepsilon(h) \bar{x}^{\prime}=\bar{c} \bar{x}^{\prime} \neq 0$, where $\bar{c}$ is the image of $c$ in $k$. By Maschke's theorem, there is an isomorphism of $k[(h)]$-modules: $\mathfrak{m} / \mathfrak{m}^{2} \simeq \bar{x}^{\prime} k \oplus \mathfrak{m}^{\prime} / \mathfrak{m}^{\prime 2}$, with $\mathfrak{m}^{\prime}=\mathfrak{m} / x^{\prime} R \subset R / x^{\prime} R=R^{\prime}$. Since by assumption $h$ induces the identity map on $R^{\prime}, \mathfrak{m}^{\prime} / \mathfrak{m}^{\prime 2}$ is a trivial $k[(h)]$-module, and $\bar{c}$ is a primitive $m$-th root of unity, $m$ being the order of $h$. In particular, $c \neq \equiv 1$ modulo $m$, which permits to conclude from the equality $h\left(x^{\prime}\right)-x^{\prime}=(c-1) x^{\prime}$ that $x^{\prime} \in \mathfrak{a}_{h}$. Now all our claims have been proved.

The next two lemmas will not be needed before the last section.

For a pseudo-reflection $h$ contained in $H$ we denote by $B(h)$ the reflecting hyperplane $\operatorname{Ker}(\varepsilon(h)-1) \subset \mathfrak{m} / \mathfrak{m}^{2}$, and set $C(h)=\operatorname{Im}(\varepsilon(h)-1) \subset \mathfrak{m} / \mathfrak{m}^{2}$. It is well known that the subgroup $H_{B}$ of those elements of $H$ which leave pointwise fixed a hyperplane $B$ is either trivial, or is cyclic generated by a pseudo-reflection: as a consequence of Maschke's Theorem, the canonical homomorphism $H_{B} \rightarrow G L_{k}\left(\left(\mathrm{~m} / \mathrm{m}^{2}\right) / B\right)$ is injective.

(13) For two pseudo-reflections $h_{1}, h_{2}$ contained in $H$, the following conditions are equivalent:

(a) $B\left(h_{1}\right)=B\left(h_{2}\right)$;

(b) $H_{B\left(h_{1}\right)}=H_{B\left(h_{2}\right)}$;

(c) $\mathfrak{a}_{h_{1}}=\mathfrak{a}_{h_{2}}$;

(d) $C\left(h_{1}\right)=C\left(h_{2}\right)$.

Proof. (a) $\Rightarrow(\mathrm{b})$ is obvious.

(b) $\Rightarrow$ (c): Let $h$ be a pseudo-reflection, generating $H_{B\left(h_{1}\right)}=H_{B\left(h_{2}\right)}$. It 
suffices to prove that for any integer $i$ between one and the order of $h$, $\mathfrak{a}_{h^{i}}=\mathfrak{a}_{h^{\prime}}$. Since $h^{i}(a)-a=\sum_{j=0}^{i-1}\left(h\left(h^{j}(a)\right)-h^{j}(a)\right) \in \mathfrak{a}_{h}$, one has $\mathfrak{a}_{h^{i}} \subset \mathfrak{a}_{h}$, in particular $x_{h^{i}}=y_{i} x_{h^{\prime}}$. Assuming $y_{i} \in \mathfrak{m}$ one gets $x_{h^{i}} \in \mathfrak{m}^{2}$, contradicting (12), hence $y_{i} \notin \mathfrak{m}$ and $\mathfrak{a}_{h^{i}}=\mathfrak{a}_{h}$.

(c) $\Rightarrow$ (d) is clear, since $C\left(h_{q}\right)=\operatorname{Im}\left(\varepsilon\left(h_{q}\right)-1\right)=\left(\mathfrak{a}_{h_{q}}+\mathfrak{m}^{2}\right) / \mathfrak{m}^{2}(q=1,2)$.

(d) $\Rightarrow$ (a): Let $H^{\prime}$ be the subgroup of $H$ generated by $h_{1}$ and $h_{2}$. By assumption, the line $C=C\left(h_{1}\right)=C\left(h_{2}\right)$ is $H^{\prime}$-stable. By Maschke's theorem, there exists a $H^{\prime}$-stable complementary hyperspace $B$. The restriction of $h_{1}$ to $B$ has $\operatorname{dim} B$ eigenvalues equal to 1 , in particular $h_{1}$ fixes a non-zero eigenvector in $B$. The existence in $B$ of a $H^{\prime}$-stable subspace complementary to the line passing through this vector permits to conclude by induction that $\left.h_{1}\right|_{B}$ is the identity, hence $B=B\left(h_{1}\right)$. By symmetry one has $B=B\left(h_{2}\right)$, hence the claim.

(The last implication is usually proved for $k=C$ by using the existence of an invariant hermitian scalar product: cf. e.g. [Sp, (4.2.15)]; the elegant argument above has been communicated to me by V. Drensky,"and replaces an earlier one worked out jointly with P. Siderov.)

When $h$ is a pseudo-reflection, then so is $g h g^{-1}$ for every $g \in H$, and $g\left(\mathfrak{a}_{h}\right)=\mathfrak{a}_{g_{h g^{-1}}}$. This defines a left action of $H$ on the set of ideals $\mathscr{A}=$ $\left\{\mathfrak{a}_{h}\right\}_{h \in \mathscr{F}}$, where $\mathscr{P}$ denotes the set of all pseudo-reflections contained in $H$.

(14) Suppose $H$ is generated by pseudo-reflections, and let $\mathscr{R} \subset \mathscr{P}$ be a generating set. Then the map $\mathscr{R} \rightarrow \mathscr{A} \mid H$, sending each reflection to the orbit of $\mathfrak{a}_{h}$, is surjective.

Proof. In the notation of the previous lemma, choose for each $h \in \mathscr{P}$ a generator $e_{h}$ of $C(h)$. For an orbit $\mathcal{O}$ of the action of $H$ on the set $\mathscr{C}$ $=\{C(h)\}_{h \in \mathcal{F}}\left(g C(h)\right.$ being $\left.C\left(g h g^{-1}\right)\right)$, set $e_{o}=\prod_{C\left(h^{\prime}\right) \in \epsilon_{o}} e_{h^{\prime}}$, which is an element of the symmetric algebra $S$ of the $k$-vector space $\mathfrak{m} / \mathfrak{m}^{2}$. By [Sp, (4.3.4.i)], $h\left(e_{\diamond}\right)=e_{\diamond}$, when $C(h) \notin \mathcal{O}$, and $h\left(e_{\odot}\right)=\operatorname{det}(h) e_{\diamond}$ otherwise. (Springer states his theorem for $k=C$ and for the action of $H$ on the dual vector space, but only notational changes are needed to transport the proof to the situation considered here.) Assume the orbit $\mathcal{O}$ is not in the image of the natural map $\mathscr{R} \rightarrow \mathscr{C} / H$, assigning to $h$ the orbit of $C(h)$. Then $h\left(e_{0}\right)=e_{0}$ for all $h \in \mathscr{R}$, and $\mathscr{R}$ being a generating set this implies $g\left(e_{0}\right)=e_{0}$ for all $g \in H$. This contradicts the fact that $e_{0}$ is not invariant, hence $\mathscr{R} \rightarrow \mathscr{C} \mid H$ is surjective. It remains to note that by (13) the map $\varphi: \mathfrak{a}_{h} \mapsto\left(\mathfrak{a}_{h}+\mathfrak{m}^{2}\right) / \mathfrak{m}^{2}$ $=C(h)$ is a bijection from $\mathscr{A}$ to $\mathscr{C}$, which commutes with the action of 
$H$, and that $\mathscr{R} \rightarrow \mathscr{C}$ factors through $\varphi$.

We conclude this section with a quotation from N. Bourbaki: it contains these consequences of his extension of Chevalley's theorem, which will be needed in the following sections.

(15) Choosing a $k$-basis $X_{1}, \cdots, X_{\ell}$, identify the symmetric algebra $S$ of the vector space $\mathrm{m} / \mathrm{m}^{2}$ with the polynomial ring $k\left[X_{1}, \cdots, X_{\ell}\right]$. If $H$ is generated by pseudo-reflections, then:

(i) $S_{+}^{H}$ is generated by $\ell$ homogeneous polynomials $F_{1}, \cdots, F_{\ell}$ which form an $S$-regular sequence, and whose degrees $d_{1}, \cdots, d_{\ell}$ satisfy the relations: $\operatorname{dim}_{k} S / S_{+}^{H} S=d_{1} d_{2} \cdots d_{\ell}=|H|$;

(ii) let $h_{1}, \cdots, h_{r}$ be the distinct pseudo-reflections contained in $H$, and let $e_{i} \in \mathfrak{m} / \mathfrak{m}^{2}$ be a generator of $\operatorname{Im}\left(\varepsilon\left(h_{i}\right)-1\right), 1 \leqslant i \leqslant r$; then the element $e=$ $e_{1} e_{2} \cdots e_{r} \in S$ is a non-zero scalar multiple of the Jacobian $J=\operatorname{det}\left(\partial F_{p} / \partial X_{q}\right)$.

References: that the generators of $S_{+}^{H}$ form a maximal $S$-regular sequence is seen from $\left[\mathrm{Bo}_{2}\right.$, Theorem 4 and Lemma 5]; since by the same theorem $S$ is free as an $S^{H}$-module, the expressions for the product of the $d_{i}$ 's are obtained by computing rank $S$ via localization at all non-zero elements and via reduction modulo $S_{+}^{H}$; (ii) is taken from $\left[\mathrm{Bo}_{2}\right.$, Proposition 6(ii)].

\section{§4. Proof of Theorem (2)}

Since $R$ is finitely-generated over $R^{H}$ by (8.ii), it follows from (11) and (15.i) that it can be generated by $|H|$ elements. Let $f: F \rightarrow R$ be a surjective homomorphism of $R^{H}$-modules, with $F$ free of rank $|H|$. Denote by $U$ the set of non-zero divisors of $R$ and let $V=\left\{N_{G}(u) \mid u \in U\right\}$. Under the hypotheses of the theorem, (6) shows that $V^{-1} R$ is $V^{-1} R^{H}$-free of rank $|H|$, hence $V^{-1} f$ is an isomorphism, being a surjective map of free modules of the same rank. This implies that $V$ meets every prime in $\operatorname{Ass}_{R^{H}}(\operatorname{Ker} f)$ $\subset$ Ass $R^{H}$. Since by definition $V$ consists only of non-zero divisors, this is possible only when $\operatorname{Ker} f=0$, i.e. when $R$ is $R^{H}$-free of rank $|H|$. Combining this with (7), we see that $R$ is $R^{G}$-free of $\operatorname{rank}|G|$. Thus we have obtained (2.i'), and in doing this we have also shown that

(16) $\operatorname{gr}_{\overline{\mathrm{m}}} \bar{R} \simeq S / S_{+}^{H} S$ as graded rings and as $k[H]$-modules. Indeed, (7) shows that $\mathfrak{m}^{G} R=\mathfrak{m}^{H} R$, hence $\bar{R}=R / \mathfrak{m}^{G} R=R / \mathfrak{m}^{H} R$, and the previous discussion has established that $\operatorname{dim}_{k} \bar{R}=|H|$. Comparing with (13.i), one sees that the canonical map in (11) is an isomorphism, which gives the 
claim of (16), and also that $\operatorname{~gr}_{\bar{\pi}} R$ is a complete intersection. It is well known that $\bar{R}$ itself is in such a case a complete intersection, (e.g. [Wk Lemma 10]), and this completes the proof of (2.ii).

The idea of the proof of (2.i) is taken from Goto's argument in $\left[\mathrm{G}_{2}\right.$, (5.1), Proof of $(3) \Rightarrow(1)]$. First note that $\sum a_{g} g \mapsto \sum a_{g}$ defines a map of left $R * G$-modules $R * G \rightarrow R$, which $R * G$-linearly splits the map $x \mapsto x\left(t_{G} r\right)$, where $r \in R$ is an element such that $t_{G}(r)=1$ : cf. (9). This shows $R$ is $R * G$-projective. But $R * G$ being $R^{G}[G]$-free of finite rank by the part of the theorem already proved, we see that $R$ is $R^{G}[G]$-projective of finite rank. According to [Ba, Chapter XI, (5.1)], in order to prove that $R$ and $R^{G}[G]$ are isomorphic as $R^{G}[G]$-modules, it is sufficient to establish a corresponding isomorphism of their localizations at some multiplicatively closed subset of $R^{G}$, consisting of non-zero divisors. This is given by the last statement of (6), taking as above $U$ to be the set of all non-zero divisors of $R$.

\section{§5. Proof of Theorem (4)}

As noted before the statement of the Theorem, the Noether, Kähler, and Dedekind differents of $R$ over $R^{G}$ coincide, and are a principal ideal, say $\mathfrak{S}\left(R \mid R^{G}\right)=j R$. The extension $R^{G} \longrightarrow R^{H}$ being unramified by (7), the modules of Kähler differentials of $R$ over $R^{a}$ and over $R^{a}$ are canonically isomorphic, which by the definition of the Kähler different (e.g. $\left[\mathrm{SS}_{1}, \S 15\right]$ ) implies that $\mathfrak{D}\left(R \mid R^{G}\right)=\mathscr{D}\left(R \mid R^{H}\right)$. Hence, in computing the different, we shall assume $G=H$. This computation is carried out in five steps.

Step 1. $j$ is a non-zero divisor, $j R$ is an $H$-stable ideal, and $j \bar{R}=$ $(0: \overline{\mathfrak{m}})_{\bar{R}}$.

For the first fact, with $U$ denoting the set of non-zero divisors of $R$, note that by (6) $U^{-1} R$ is unramified over $V^{-1} R^{H}$, which clearly implies $U^{-1}(j R)=U^{-1} R$ : cf. [ $\mathrm{SS}_{1},(15.2)$ and (15.3)].

In order to prove that $j R$ is $H$-stable, note that the multiplication map $\mu: R \otimes_{R^{H}} R \rightarrow R, \mu(a \otimes b)=a b$, becomes a homomorphism of $H$-modules, if one defines $h(a \otimes b)=h(a) \otimes h(b)$. This implies $I=\operatorname{Ker} \mu$ is $H$-stable, hence also its annihilator $(0: I)$ in $R \otimes_{R^{H}} R$ is $H$-stable. By definition, the Noether different of $R$ over $R^{H}$ is the ideal $\mu(0: I)$, hence the stability of $j R$ is established.

For the proof of the last assertion, we note that by the base change properties of the different $\left[\mathrm{SS}_{1},(15.1)\right], \mathfrak{S}(\bar{R} \mid k)=\mathscr{S}\left(R \mid R^{H}\right) \bar{R}=j \bar{R}$. On the 
other hand, by (2), the $k$-algebra $\bar{R}$ is an artinian complete intersection, with residue field $k$, and with $\operatorname{dim}_{k} \bar{R}=|H|$ prime to the characteristic of k. A result of Scheja and Storch ([SS, p. 187], or $\left[\mathrm{SS}_{1}\right.$, p. 101]) says that under these conditions $\mathfrak{D}(\bar{R} \mid k)=(0: \overline{\mathfrak{n}})_{\bar{R}}$, and we are done.

It follows from Step 1 that for each $g \in H$ there is a uniquely defined $\chi(g) \in R^{\times}$, such that $g(j)=\chi(g) j$. It is well-known and easily verified, that the map $\chi: H \rightarrow R^{\times}, g \mapsto \chi(g)$, is a crossed homomorphism: $\chi\left(g g^{\prime}\right)=$ $g\left(\chi\left(g^{\prime}\right)\right) \cdot \chi(g)$.

Step 2. The set $R_{x}^{H}=\{a \in R \mid \forall g \in H: g(a)=\chi(g) \cdot a\}$ of semiinvariants of $H$ of weight $\chi$ is a free $R^{H}$-module generated by $j$.

Following Hinič [X], we consider the twisted Reynolds operator $a \mapsto$ $\rho_{\chi}(a)$, where $\rho_{\chi}=|H|^{-1} \sum_{g \in H}(\chi(g))^{-1} g \in R * H$. Since it gives an $R^{H}$-linear splitting of the inclusion $R_{x}^{H} \longleftrightarrow R, R_{x}^{H}$ is a free $R^{H}$-module by (2.i'). Denoting by bars reduction modulo $\mathrm{m}^{H}$, the naturality of $\rho_{z}$ shows that $R_{\chi}^{H} \otimes_{R^{H}} k$ is the direct summand $\bar{R}_{\bar{\chi}}^{H}$ of $\bar{R}$ corresponding to the crossed homomorphism $\bar{\chi}: H \rightarrow R^{\times} \rightarrow k^{\times}$. Since $H$ acts trivially on $k, \bar{\chi}$ is simply a linear character (or group homomorphism $H \rightarrow k^{\times}$), and by Maschke's theorem $\bar{R}_{\bar{\chi}}^{H}$ is as a $k[H]$-module the semi-simple component of $\bar{R}$ which corresponds to the linear representation defined by $\bar{\chi}$. By (2.i) $\bar{R}$ is equivalent to the regular representation, in which every linear representation enters with multiplicity one. Hence $\bar{R}_{\bar{z}}^{H} \simeq k$, which implies $R_{x}^{H} \simeq R^{H}$ as $R^{H}$-modules. Finally, $R_{x}^{H}$ being a direct summand of $R$, in order to prove that it is generated by $j$ it is sufficient to show that $j$ has a non-zero image in $\bar{R}$, and this is given by the last claim of the previous step.

The next proposition gives a remarkable ideal-theoretic characterization of $\mathfrak{D}\left(R \mid R^{H}\right)$ :

Step 3. $j R$ is the intersection of all the $H$-stable ideals of $R$, which are not contained in $\mathrm{m}^{H} R$.

One inclusion being clear by Step 1 , we denote by $\mathfrak{b}$ an $H$-stable ideal, $\mathfrak{b} \not \subset \mathfrak{m}^{H} R$, and want to show that $j \in \mathfrak{b}$. First note that $j$ is contained in $\mathfrak{b}+\mathfrak{m}^{H} R$. Indeed, $\bar{R}$ being an artinian complete intersection by (2.i), the ideal $\mathfrak{b} \bar{R} \neq 0$ contains the socle $(0: \overline{\mathfrak{m}})_{\bar{R}}$, which by Step 1 is equal to $j \bar{R}$.

Write $j=b+\sum m_{i} a_{i}$ with $b \in \mathfrak{b}, m_{i} \in \mathfrak{m}^{H}, a_{i} \in R$. Then:

$$
j=\rho_{\chi}(j)=\rho_{x}(b)+\sum m_{i} \rho_{x}\left(a_{i}\right) .
$$

By Step 2 we can write $\rho_{\chi}\left(a_{i}\right)=j c_{i}$ with $c_{i} \in R^{H}$, hence:

$$
j\left(1-\sum m_{i} c_{i}\right)=\rho_{x}(b) \in \mathfrak{b},
$$


the inclusion holding since $\mathfrak{b}$ is $H$-stable. We conclude that $j \in \mathfrak{b}$, which proves our claim.

Step 4. $x R$ is an $H$-stable ideal, and $x \bar{R}=(0: \overline{\mathfrak{m}})_{\bar{R}}$.

Recall that $x=x_{1} x_{2} \cdots x_{r}$, where $x_{i}$ is an element of $\mathfrak{m}$ defined by the pseudo-reflection $h_{i}$ via (12) (or (1)), and $h_{1}, h_{2}, \cdots, h_{r}$ are all the distinct pseudo-reflections contained in $H$. By (12) again, $R x_{i}$ is the ideal $\mathfrak{a}_{i}$, generated by $h_{i}(a)-a$ as a runs through $R$, hence $x R=\mathfrak{a}_{1} \mathfrak{a}_{2} \cdots \mathfrak{a}_{r}$. Now clearly for any $g \in H$ and any pseudo-reflection $h \in H$, $g h g^{-1}$ is a pseudo-reflection. Since $a_{g h g-1}=g\left(a_{h}\right)$ by a direct computation, the previous remarks show that $x R$ is $H$-stable.

Let now $d$ be the largest integer for which $\overline{\mathfrak{m}}^{d} \neq 0$, and consider the inclusions:

$$
(0: \overline{\mathfrak{m}})_{\overline{\mathfrak{R}}} \supset \overline{\mathfrak{m}}^{d}=\mathrm{gr}_{\overline{\mathfrak{m}}}^{d} \bar{R} \subset\left(0: \mathrm{gr}_{\overline{\mathrm{m}}} \overline{\mathfrak{m}}\right)_{\mathrm{gr}_{\overline{\mathrm{m}}}} \bar{R},
$$

where the equality denotes a canonical identification. Since by (2.ii) the vector spaces on both ends are one-dimensional, we have equalities throughout. Hence in order to prove the equality $x \bar{R}=(0: \overline{\mathfrak{m}})_{\bar{R}}$ it is sufficient to show that the socle of $\operatorname{gr}_{\overline{\mathrm{m}}} \bar{R}$ is generated by the initial form $\bar{x}$ of the image of $x$ in $\bar{R}$. Note that $\bar{x}$ is the product of $\bar{x}_{1}, \bar{x}_{2}, \cdots, \bar{x}_{r}$, where $\bar{x}_{i}$ is the leading form of the image of $x_{i}$ in $\bar{R}$. By (12) $\bar{x}_{i}$ has degree one and is a generator of the image in $\overline{\mathfrak{m}} / \overline{\mathfrak{m}}^{2}$ of the vector space $\operatorname{Im}\left(\varepsilon\left(h_{i}\right)-1\right) \subset \mathfrak{m} / \mathfrak{m}^{2}$.

Setting $\bar{S}=S / S_{+}^{H} S$ and denoting by bars the images in $\bar{S}$ of the homogeneous elements of $S$, we identify $\bar{S}$ with $\mathrm{gr}_{\mathrm{m}} \bar{R}$ as in (16). Under this identification, the preceding discussion yields the equality $\bar{x}=\bar{e}_{1} \bar{e}_{2} \cdots \bar{e}_{r}$, where the $e_{i}$ 's are the elements of $S_{1}$ defined in (15.ii). According to this proposition, we are reduced to showing the equality $J \bar{S}=\left(0: \bar{S}_{+}\right)_{\bar{S}}$, with $J$ denoting the Jacobian.

This can be obtained by base change, as in the proof of the last statement of Step 1, by invoking the description of the different $\mathfrak{S}\left(S \mid S^{H}\right)$ given in [ $\mathrm{Bo}_{2}$, Proposition 6(i)]. A simple alternate argument is as follows. Let $K$ denote the Koszul complex on the images of $X_{1}, \cdots, X_{\ell}$ in $\bar{S}$, and choose a basis $T_{1}, \cdots, T_{\ell}$ of $K_{1}$ such that $\mathrm{d} T_{i}=X_{i}$ modulo $S_{+}^{H} S$. From (15.i) the $d_{i}$ 's are invertible in $k$, hence by Euler's formula the classes of the cycles $z_{i}=d_{i}^{-1} \sum_{q=1}^{\ell}\left(\partial F_{i} / \partial X_{q}\right) T_{q}$ form for $i=1, \cdots, \ell$ a basis of $H_{1}(K)$. The artinian ring $\bar{S}$ being complete intersection (13.i), $H(K)$ is the exterior algebra on $H_{1}(K)$, in particular $H_{\ell}(K)=J \cdot \bar{S} T_{1} \wedge \cdots \wedge T_{\ell}$. Since the map 
$\bar{S} \rightarrow K_{\ell}$, which sends 1 to $T_{1} \wedge \cdots \wedge T_{\ell}$, induces an isomorphism of $\left(0: \bar{S}_{+}\right)_{\bar{S}}$ with $H_{\ell}(K)$, the proof of Step 4 is now complete.

Step 5. Conclusion of the proof. By Step $4 x R$ is an $H$-stable ideal, which is not contained in $\mathrm{m}^{H} R$, hence $j=x y$ for some $y \in R$, as follows from Step 3. Assuming $y \in \mathfrak{m}$, Step 4 implies the image of $j$ in $\bar{R}$ is 0 , which contradicts Step 1 . We see that $y \in R^{\times}$, which implies the required equality $j R=x R$.

Now we turn to the description of the discriminant. By $\left[\mathrm{SS}_{2},(5.4)\right]$, $\mathfrak{D}\left(R \mid R^{G}\right)$ is generated by the norms of the elements of $\mathfrak{D}\left(R \mid R^{G}\right)$, hence by $N_{R \mid R^{G}}(x)$. One has to show that the norm of an element $a \in R$, i.e. the determinant of the $R^{a}$-linear map $b \mapsto a b, b \in R$, is equal to $N_{G}(a)=\prod_{g \in G} g(a)$. But $N_{R \mid R^{H}}=N_{H}: R \rightarrow R^{H}$ by $\left[\mathrm{SS}_{1},(21.14)\right]$, since $|H| \in R^{\times}$, and $N_{R^{H} \mid R^{G}}=$ $N_{G / H}: R^{H} \rightarrow R^{G}$ by $\left[\mathrm{SS}_{1},(21.2)\right]$, since $R^{H}$ is a Galois extension of $R^{G}$ (7). By the transitivity of norms one now gets $N_{R \mid R^{G}}=N_{R^{H} \mid R^{G}} \cdot N_{R \mid R^{H}}=N_{G / H} \cdot N_{H}$ $=N_{G}$.

Note on the proof. A close look at the proof of Theorem (4) shows that from the statement of (15.ii), only the fact that $e$ and $J$ have the same degree is used, and this boils down to the expression $\sum_{i=1}^{\ell}\left(d_{i}-1\right)$ for the number of pseudo-reflections contained in $H$. When the characteristic of $k$ is zero, this is an easy consequence of Molien's Theorem (cf. [ $\mathrm{Bo}_{2}$, Proposition 3]), hence in this case our result gives an independent computation of the different $\mathfrak{D}\left(S \mid S^{H}\right)$ for the polynomial ring. In contrast, when the characteristic of $k$ is positive, Bourbaki's proof of (15.ii) is based on the knowledge of $\mathfrak{S}\left(S \mid S^{H}\right)$.

\section{§6. Generalized reflections.}

The main result of this section, besides clarifying the relations of the work above to that of some other authors, can be viewed as a criterion for checking whether the premises of Theorems (2) and (4) are met. Recall that a generalized reflection in the sense of Hochster and Eagon [HE] is a non-identical automorphism of $R$, which is contained in the inertia subgroup of some principal ideal, generated by a regular element of $\mathrm{m}$.

(17) THEOREM. Let $G$ be a finite group of automorphisms of the local ring $(R, \mathfrak{m}, k)$, such that the order of $H=G^{T}(\mathfrak{m})$ is invertible in $k$. Consider the following conditions: 
(a) $H$ is generated by pseudo-reflections, and $G$ acts on $R$ generically without inertia;

(b) $H$ is generated by generalized reflections.

Then (a) implies (b).

If furthermore $R$ contains some field, then the converse also holds.

Until the end of this section we assume $|H| \in R^{\times}$. We start by a lemma, which immediately reduces everything to the proof that condition (b) implies that $G$ acts on $R$ generically without inertia.

(18) (i) Each generalized reflection is a pseudo-reflection.

(ii) Assume $G$ acts on $R$ generically without inertia. Then each pseudoreflection is a generalized reflection.

Proof. (i) is a consequence of the easy implication (b) $\Rightarrow$ (a) of (12).

(ii) According to (12), the pseudo-reflection $h$ lies in the inertia subgroup of a non-zero principal ideal $x_{h} R$. If $x_{h}$ were a zero divisor, there would be a prime $\mathfrak{P} \in \operatorname{Ass}(R)$ such that $x_{h} R \in \mathfrak{P}$, hence $G^{T}(\mathfrak{P}) \supset G^{T}\left(x_{h} R\right) \ni$ $h \neq 1$, which contradicts our hypothesis.

The rest of the proof of Theorem (17) is subdivided into three steps, only the last one of which makes use of the equi-characteristic assumption. Condition (b) is assumed.

Step 1. Every pseudo-reflection contained in $H$ is a generalized reflection.

In fact, by our hypothesis and by (18.i), $H$ is generated by a set $\mathscr{R}$ of pseudo-reflections, such that $\mathfrak{a}_{h^{\prime}}$ is a regular principal ideal for each $h^{\prime} \in \mathscr{R}$. Let $\mathscr{P}$ be the set of all pseudo-reflections of $H$. According to (14) for each $h \in \mathscr{P}$ there is an $h^{\prime} \in \mathscr{R}$ such that $\mathfrak{a}_{h}$ and $\mathfrak{a}_{h^{\prime}}$ are in the same orbit for the natural action of $H$, i.e. there is a $g \in H$ such that $a_{h}=g\left(a_{h^{\prime}}\right)$. This shows that $a_{h}$ is generated by a regular non-invertible element of $R$, hence $h$ is a generalized reflection.

Step 2. Reduction to the case of complete $R$.

Let $a_{h}(R)$ denote the ideal generated by the elements $h(a)-a$, when $a$ ranges over $R$. The argument in the proof that (12.a) implies (12.c) shows that $\mathfrak{a}_{h}(\hat{R})=\mathfrak{a}_{h}(R) \hat{R}$, where the action of $G$ on $\hat{R}$ is that described in (10.ii). Since $H$ clearly is contained in $G^{T}(\mathfrak{m} \hat{R})$, the equality shows, for one thing, that $H$ considered as a subgroup of $\operatorname{Aut}(\hat{R})$ is generated by generalized reflections. Assuming that the fact that condition (b) of the 
Theorem has already been shown to imply for complete rings that $G$ acts generically without inertia, we shall deduce the corresponding property for $R$. So let $\mathfrak{\beta}$ be an associated prime of $R$ and $h$ be an arbitrary element of $G^{T}(\mathfrak{\beta})$. Choose an associated prime $\mathfrak{Q}^{\prime}$ of $k(\Re) \otimes_{R} \hat{R}$, and let $\mathfrak{Q}$ be its inverse image in $\hat{R}$ for the canonical map. Then $\mathfrak{\cap} R=\mathfrak{R}$, and $\mathfrak{Q}$ $\in \operatorname{Ass}(\hat{R})$. By our choices,

$$
\mathfrak{a}_{h}(R)=\mathfrak{a}_{h}(R) \hat{R} \subset \mathfrak{\beta} \hat{R} \subset \mathfrak{Q},
$$

hence $h \in G^{T}(\cong)$, and the last group is trivial by assumption.

Before going over to the last step, we recall a well-known fact:

(19) If $H$ is generated by generalized reflections, then $R$ is $R^{H}$-free of finite rank.

Finite generation being clear by (8), it remains to establish flatness, which has been done by Serre in [Se, pp. 7-11]. A slicker arrangement of his argument is given in the proof of Theorem 1 in $\left[\mathrm{Bo}_{2}\right]$, which treats the graded case. There is no trouble in translating it to the local case, once it is noted that by (12) for each reflection of $h$ there is a $x_{h} \in \mathrm{m}$ such that for an arbitrary $R$-module $M$ with compatible $G$-action, $(h-1) M \subset$ $x_{h} M$.

Step 3. Completion of the proof that (b) implies (a) in the equicharacteristic case.

By the result of Step 2 we can assume $R$ is complete, hence so is $R^{H}$ according to (10.ii). Choose a coefficient field for $R^{H}$. Since the inclusion $R^{H} \subset R$ induces an isomorphism of residue fields, this is also a coefficient field for $R$, and we shall denote it by the same letter $k$. One sees that $H$ acts on $R$ by $k$-linear automorphisms.

Now we can apply the following lifting result, proved in G. Müller's thesis [M]. In the preprint version of the present paper, circulated before the author was aware of [M], instead of a reference to this proposition we had to assume that (a statement slightly weaker than) its conclusion holds for the action of $H$ on $R$.

(20) [M, (2.3)]. Let $R=R^{\prime} / \mathfrak{a}$, where $R^{\prime}$ is the formal power series ring $k\left[\left[X_{1}, \cdots, X_{n}\right]\right], \mathfrak{m}^{\prime}=\left(X_{1}, \cdots, X_{n}\right), a \subset \mathfrak{m}^{\prime 2}$. Suppose $H$ acts on $R$ as a (finite) group of k-algebra automorphisms. Then there is a group $H^{\prime}$ of k-algebra dutomorphisms of $R^{\prime}$, such that a is $H^{\prime}$-stable, and the canonical projection $R^{\prime} \rightarrow R$ induces a commutative diagram of group homomorphisms: 


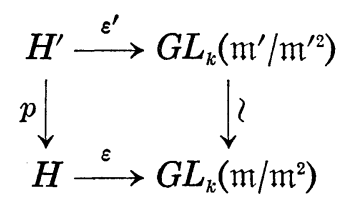

In particular, $p$ is an isomorphism (cf. e.g. (10.i)), and restricts to a bijection from the set of pseudo-reflections of $H^{\prime}$ to that of $H$. We shall henceforth identify $H$ and $H^{\prime}$ (and set $p=i d$ ).

In the notation of the previous two steps, note the equality $a_{h}(R)=$ $\mathfrak{a}_{h}\left(R^{\prime}\right) R$ for every $h \in H$, hence one has

$$
\prod \mathfrak{a}_{h}(R)=\prod \mathfrak{a}_{h}\left(R^{\prime}\right) R
$$

when $h$ ranges over the set $\mathscr{P}$ of pseudo-reflections of $H$. Theorem (4), applied to the regular domain $R^{\prime}$, shows that the product $\prod_{h \in \mathscr{P}} \mathfrak{a}_{h}\left(R^{\prime}\right)$ is generated by a non-zero element $x^{\prime}$, which is contained in every $H$-stable ideal $\mathfrak{b}^{\prime}$ of $R^{\prime}$, such that $\mathfrak{b}^{\prime} \not \subset \mathfrak{m}^{\prime H} R^{\prime}$. We conclude that $a \subset \mathfrak{m}^{\prime H} R^{\prime}$, since otherwise one would have had $x^{\prime} \in \mathfrak{a}$, implying $\prod_{h \in \mathscr{P}} \mathfrak{a}_{h}(R)=0$ in $R$; this, however, is impossible, since by the result of Step 1 and by (12) each $\mathfrak{a}_{h}$ is generated by a non-zero divisor in $R$.

Applying (9), we now see that $\mathfrak{m}^{H} R=\mathfrak{m}^{H} R=\left(\mathfrak{m}^{\prime H} R^{\prime}\right) / \mathfrak{a}$, hence $R / \mathfrak{m}^{H} R$ $\simeq R^{\prime} / \mathfrak{m}^{\prime H} R^{\prime}$. By (2) the dimension of the left-hand side over $k=R^{\prime H} / \mathrm{m}^{\prime H}$ $=R^{H} / \mathrm{m}^{H}$ is equal to $|H|$, hence by (19) $R$ is $R^{H}$-free of $\operatorname{rank}|H|$, which together with (7) shows that $R$ is $R^{G}$-free of rank $|G|$.

From (6) one now sees that the required equalities $G^{T}(\mathfrak{P})=1$ for $\mathfrak{P} \in$ Ass $(R)$ will follow once we show that $U^{-1} R$ is unramified over $V^{-1} R^{G}$, where $U$ denotes the set of all non-zero divisors of $R$. To this end consider the natural map of $R^{G}$-modules: $R^{G} \otimes_{R^{\prime} G} R^{\prime} \rightarrow R$, which is surjective since by (9) the left-hand side is $R^{\prime} / \mathfrak{a}^{G} R^{\prime}$. Also, the preceding discussion shows that $R$ is $R^{G}$-free of rank $|G|$, while the same conclusion holds for $R^{G} \otimes_{R^{\prime} G} R^{\prime}$ in view of (2). Consequently, we are in presence of a module isomorphism which, being induced by a map of rings, shows that $R^{G} \otimes_{R^{\prime}} R^{\prime} \simeq R$ as rings. By the change of base properties of the Kähler different $\left[\mathrm{SS}_{1},(15.2)\right]$ and by (4) one gets:

$$
\mathfrak{D}\left(R \mid R^{G}\right)=\mathfrak{S}\left(R^{\prime} \mid R^{\prime G}\right) R=x^{\prime} R=x R .
$$

As already noted above, $x R$ is the product of the regular principal ideals $\mathfrak{a}_{h}(R)(h \in \mathscr{P})$, so that one obtains:

$$
\mathfrak{D}\left(U^{-1} R \mid V^{-1} R^{G}\right)=U^{-1} \mathfrak{D}\left(R \mid R^{G}\right)=U^{-1} R,
$$


which establishes that $U^{-1} R$ is non-ramified over $V^{-1} R^{a}$.

The proof of Theorem (17) is now complete.

It is a pleasure to thank R. Achilles, G. Almkvist, V. Drensky, and P. Siderov for discussions of various aspects of this paper; C. Lech for explaining to me the unpublished proof of his result used in (3); and G. Müller for sending me a copy of his thesis.

\section{REFERENCES}

[A] L. L. Avramov, Homology of local flat extensions and complete intersection defects, Math. Ann., 228 (1977), 27-37.

[Ba] H. Bass, Algebraic K-Theory, W. A. Benjamin, N.-Y., 1969.

[Bo $0_{1}$ N. Bourbaki, Algèbre commutative, Chapitre V, § 2, Hermann, Paris, 1964.

$\left[\mathrm{Bo}_{2}\right]$ - Groupes et algèbres de Lie, Chapitre V, § 5,, Hermann, Paris, 1968.

[C] C. Chevalley, Invariants of finite groups generated by reflections, Amer. J. Math., 67 (1955), 778-782.

[CHR] S. U. Chase, D. K. Harrison and A. Rosenberg, Galois theory and Galois cohomology of commutative rings, Memoirs Amer. Math. Society, 52 (1965), 15-33.

[F] R. Fossum, The divisor class group of a Krull domain, Ergebnisse der Math. Bd., 74, Springer-Verlag, Berlin-Heidelberg-N.-Y., 1973.

$\left[\mathrm{G}_{1}\right] \mathrm{S}$. Goto, Invariant subrings under the action of a finite group generated by pseudo-reflections, Osaka J. Math., 15 (1978), 47-50.

[G $\left.\mathrm{G}_{2}\right]$ - The rank of syzygies under the action by a finite group, Nagoya Math. J., 71 (1978), 1-12.

[HE] M. Hochster and J. A. Eagon, Cohen-Macaulay rings, invariant theory, and the generic perfection of determinational loci, Amer. J. Math., 93 (1971), 1020-1056.

[HK] J. Herzog und E. Kunz (Hrsg.), Der kanonische Modul eines Cohen-MacaulayRings, Lecture Notes in Math., 238, Springer-Verlag, Berlin-Heidelberg-N.-Y., 1971.

[K] C. M. Knighten, Differentials on quotients of algebraic varieties, Trans. Amer. Math. Soc., 177 (1973), 65-89.

[L] C. Lech, Inequalities related to certain couples of local rings, Acta Math., 112 (1964), 69-89.

[LP] M. Lorenz and D. S. Passman, Observations on crossed products and fixed rings, Communications in Algebra, 8 (1980), 743-779.

[M] G. Müller, Endliche Automorphismengruppen analytischer C-Algebren und ihre Invarianten, Math. Ann., 260 (1982), 375-396.

[Se] J.-P. Serre, Groupes finis d'automorphismes d'anneaux locaux réguliers, Colloque d'Algèbre E.N.S.J.F., 1967.

[Si $i_{1}$ B. Singh, Invariants of finite groups acting on a local unique factorization domain, J. Indian Math. Soc., 34 (1970), 31-38.

[ $\left.\mathrm{Si}_{2}\right]$ - Q Quotient of a local singularity by a finite pseudo-reflection group, Preprint, 1980 (to appear in J. Indian Math. Soc.).

[Sp] T. A. Springer, Invariant Theory, Lecture Notes in Math. 585, Springer-Verlag, Berlin-Heidelberg-N.Y., 1977.

[SS $\left.{ }_{1}\right]$ G. Scheja und U. Storch, Lokale Verzweigungstheorie, Schriftenreihe der Mathematischen Institutes der Universität Freiburg i. Ue. Nr. 5, WS 1973/74. 
[SS $\left.{ }_{2}\right]$ - Über Spurfunktionen bei vollständigen Durchschnitten, J. Reine und Angew. Math., 278/279 (1975), 174-190.

[St] U. Storch, Über das Verhalten der Divisorenklassengruppen normaler Algebren bei nicht ausgearteten Erweiterungen und über endliche Derivationen analytischer Algebren, Habilitationsschrift Bochum, 1971.

[Wj] J. Watanabe, On Cohen-Macaulay rings with many zero divisors and applications, J. Algebra, 39 (1976), 1-14.

[Wk $\mathrm{W}_{1}$ K. Watanabe, Certain invariant subrings are Gorenstein, I, Osaka J. Math., 11 (1974), 1-8.

[Wk $\mathrm{W}_{2}$ ] - Invariant subrings of a Gorenstein ring by a finite group generated by pseudoreflections, J. Fac. Sci. Univ. Tokyo, Sec. IA, 24 (1977), 87-92.

[X] V. A. Hinič, On the Gorenstein property of the ring of invariants of a Gorenstein ring, Izvestija Akad. Nauk. SSSR, Ser. Mat., 40 (1976), 50-56 (in Russian); English translation: Math. USSR, Izvestija, 10 (1976), 47-53.

Institute of Mathematics

Bulgarian Academy of Sciences

1113 Sofia

Bulgaria 